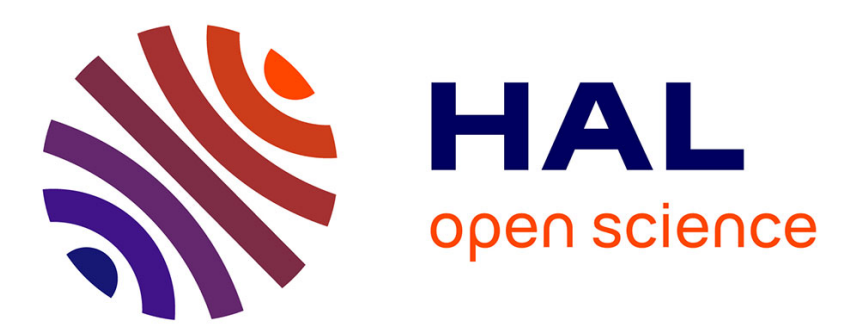

\title{
The association of acute exercise-induced ischaemia with systemic vasodilator function in patients with peripheral arterial disease
}

Majda Joras, Pavel Poredoš

\section{- To cite this version:}

Majda Joras, Pavel Poredoš. The association of acute exercise-induced ischaemia with systemic vasodilator function in patients with peripheral arterial disease. Vascular Medicine, 2008, 13 (4), pp.255262. 10.1177/1358863X08096347 . hal-00571389

\section{HAL Id: hal-00571389 \\ https://hal.science/hal-00571389}

Submitted on 1 Mar 2011

HAL is a multi-disciplinary open access archive for the deposit and dissemination of scientific research documents, whether they are published or not. The documents may come from teaching and research institutions in France or abroad, or from public or private research centers.
L'archive ouverte pluridisciplinaire HAL, est destinée au dépôt et à la diffusion de documents scientifiques de niveau recherche, publiés ou non, émanant des établissements d'enseignement et de recherche français ou étrangers, des laboratoires publics ou privés. 


\title{
The association of acute exercise-induced ischaemia with systemic vasodilator function in patients with peripheral arterial disease
}

\author{
Majda Joras and Pavel Poredoš
}

\begin{abstract}
Patients with peripheral arterial disease (PAD) frequently experience ischaemic attacks of the affected tissues during exercise. The present study assesses the association of transient exercise-induced leg ischaemia with vasodilator function of the clinically unaffected brachial artery over the course of 4 hours. Thirty male patients with symptomatic PAD and 14 age- and sex-matched healthy controls were included in the study. They performed a treadmill exercise until intolerable exerciseinduced ischaemic pain occurred in the affected lower extremity, or for at most 10 min. Flow-mediated dilation (FMD) of the brachial artery was measured at baseline, 30 minutes, 2 hours and 4 hours after exercise. Baseline FMD values were significantly diminished in patients $(7.03 \pm 1.99 \%$ vs $8.22 \pm 1.60 \%$ in controls, $p=0.009)$. A significant decrease in FMD was observed in patients after exercise (at 30 minutes: $3.92 \pm 1.78 \%$ vs $7.03 \pm 1.99 \%$ at baseline, $p<0.001$; at 2 hours: $6.36 \pm 2.12 \%$ vs $7.03 \pm$ $1.99 \%$ at baseline, $p=0.005)$, followed by a gradual return to its baseline value, whereas FMD in controls non-significantly increased after exercise. The difference in the pattern of FMD change over time between patients and controls was significant $(p<0.001)$. This study shows that in PAD patients ischaemia during intermittent claudication is related to a transitory functional deterioration of the distant arteries. This indicates the harmful systemic effects of repeated ischaemic attacks during exercise and might explain the generalized and advanced nature of atherosclerotic disease in PAD patients.
\end{abstract}

Key words: exercise; flow-mediated vasodilation; ischaemia; peripheral arterial disease; vasodilator function

\section{Introduction}

Many patients with peripheral arterial disease (PAD) experience ischaemic attacks in hypoperfused tissues during physical activity - intermittent claudication. In these patients, atherosclerotic disease has been observed to be more advanced and generalized than in healthy individuals of comparable ages. ${ }^{1,2}$ But exact mechanisms leading to enhanced atherosclerosis in PAD patients have not been well explained. Some studies have shown increased levels of pro-inflammatory cytokines in the circulation and additional deterioration of systemic vasodilator function shortly after intermittent claudication in PAD patients. ${ }^{3-6}$ These findings support the concept of harmful mediators repeat-

Department for Vascular Medicine, University Medical Centre, Ljubljana, Slovenia

Correspondence to: Majda Joras, Department for Vascular Medicine, University Medical Centre, Zaloška 7, SI-1525 Ljubljana, Slovenia.Email: majda_joras@yahoo.com edly released from peripheral tissues to the circulation after each exercise-induced ischaemic episode, with the effects exceeding their initial local nature. On the contrary, however, other studies indicate the long-term beneficial effects of physical activity on systemic vasodilator function, ${ }^{7}$ on reducing cardiovascular events and mortality in PAD patients ${ }^{8}$ and on improvement of the exercise capability of PAD patients. ${ }^{9}$

Impaired vascular function is considered to be a risk factor for the development of atherosclerosis and cardiovascular events. ${ }^{10}$ It is present in all stages of PAD, is already in asymptomatic patients, and is related to the severity of the disease..$^{11}$ One of the most widely used and until now well-recognized methods of vascular (dys)function assessment, is measurement of the capability of endotheliumdependent dilation of arteries during the provocation of nitric oxide (NO) release. It is diminished when reduced amounts of vasodilatory substances are released from the functionally damaged endothelial cells, above all NO. ${ }^{12,13}$ The capability of 
vasodilation depends not only on endothelial function, but also on vascular smooth muscle function. However, in patients with atherosclerotic vascular disease and/or risk factors, endothelial function is particularly impaired, while vascular smooth muscle function is principally unaffected. ${ }^{11,14}$

To date, there are no published data on the duration of changes and vasodilator function in PAD patients after intermittent claudication. Therefore, the aim of this study was to assess the time course of changes in vasodilator function of the clinically unaffected brachial artery after acute exerciseinduced leg ischaemia in PAD patients. Since vasodilatory response of the conduit peripheral arteries might be provoked by an increased blood flow, ${ }^{12}$ vasodilator function was investigated during reactive hyperaemia in the brachial artery and the term flow-mediated vasodilation (FMD) is used hereafter in this study.

\section{Materials and methods}

\section{Participants and study protocol}

Thirty patients with intermittent claudication (all males, median age (interquartile range): 60.6 (54.9-62.6) years, range 44.5-65.4 years) and 14 age- and sex-matched healthy volunteers (age 57.8 (52.4-63.0) years, range 45.1-65.2 years) were included. Patients were recruited to the study from the Department for Vascular Medicine at the University Medical Centre in Ljubljana, after exclusion of symptomatic coronary artery disease and cerebrovascular disease in the past year. They met the clinical criteria for stage II of the Fontaine classification, ${ }^{15}$ with the symptoms lasting at least 6 months. Except for intermittent claudication, they had no other exercise-limiting factors. All had stable PAD (the absence of disease deterioration, according to their medical reports) and no acute vascular event in the last 3 months before inclusion in the study. One patient had a history of clinically manifest coronary artery disease in the past. Participants had not been exposed to cigarette smoke for at least 6 months. Exclusion criteria were: diabetes, heart failure, atrial fibrillation, renal or liver disease, or inflammatory disease of any aetiology. All patients were receiving acetylsalicylic acid (100 mg daily), and some of them additionally pentoxifylline (1200 $\mathrm{mg}$ daily) and/or statin (simvastatin or atorvastatin, different daily doses). None of the patients had received any antihypertensive/vasoactive or other anti-inflammatory medications for at least 14 days, or any invasive treatment in the last 6 months before the study. After the exclusion of contraindications, acetylsalicylic acid (100 mg daily) was temporarily introduced in healthy control participants a week before the examinations. At least 2 days before the examinations, the participants were not exposed to significant physical exercise. Examinations were performed in the morning, after a 12-hour overnight fast, including from caffeine. All participants gave their informed consent to take part in the study, approved by the Slovene National Ethics Committee.

After taking their medical history and a brief physical examination, body height and weight were measured and body mass index (BMI) calculated (weight $[\mathrm{kg}] / \mathrm{height}^{2}[\mathrm{~m}]$ ). Systolic and diastolic blood pressures were recorded and ankle systolic pressure was measured in both legs with a Doppler ultrasound probe. The measurements of brachial and ankle systolic pressures were repeated immediately and $5 \mathrm{~min}$ after exercise. On each occasion, the ankle-brachial index (ABI) was calculated for both legs and the lower value used in further analysis.

Blood samples were taken at the beginning of the study, after a 20 -min rest, from the left antecubital vein. Patients performed exercise - a constant load treadmill test $\left(12^{\circ}\right.$ slope, $\left.3.2 \mathrm{~km} / \mathrm{h}\right)$ - also continuing after the onset of ischaemic pain, until the pain in the affected lower extremity became intolerable. Then the walking distance up to the onset of intermittent claudication and the maximal walking distance were calculated. Control participants exercised under the same protocol for $10 \mathrm{~min}$. The measurements of FMD were performed before exercise and then $30 \mathrm{~min}, 2 \mathrm{~h}$ and $4 \mathrm{~h}$ after exercise.

\section{Ultrasound examinations}

In all participants, non-invasive ultrasound studies of vascular function were performed using the standard $10-\mathrm{MHz}$ linear array transducer of a highresolution ultrasound system (ATL Ultrasound, Bothell, USA).

Investigation of vascular function was based on the technique described by Celermajer, et al. ${ }^{16}$ The right brachial artery diameter proximal to the antecubital fossa and blood flow velocity were measured in the longitudinal plane at rest and during reactive hyperaemia. Reactive hyperaemia was achieved by rapid release of a pneumatic pressure cuff placed around the forearm and inflated to a suprasystolic pressure for $5 \mathrm{~min}$. Blood flow velocity was measured by a mid-artery pulsed Doppler signal at an angle of $68^{\circ}$ to the vessel, obtained at rest and during reactive hyperaemia $15 \mathrm{~s}$ after forearm cuff deflation. Brachial artery flow for both study conditions was calculated from blood flow velocity and arterial diameter. Measurements of brachial artery diameter were performed at the end of diastole, which was determined by simultaneous monitoring of the electrocardiogram. Brachial artery diameter was measured by an independent observer, at a 
fixed distance from a selected anatomic marker. Each measurement was performed three times, recorded and then averaged. FMD was expressed as the change in the end diastolic brachial artery diameter during reactive hyperaemia $60 \mathrm{~s}$ after forearm cuff deflation compared to its baseline value.

\section{Blood sampling and analyses}

Blood was sampled from the left antecubital vein, using a tourniquet applied for $1 \mathrm{~min}$ or less. No blood additives were used for basic haematological and biochemical examinations. For the analysis of fibrinogen and oxidatively modified low-density lipoprotein (ox-LDL), blood was allowed to flow directly into a siliconized glass vacuum tube containing $0.13 \mathrm{~mol} \mathrm{l}^{-1}$ trisodium citrate. Immediately after sampling, the blood was centrifuged for $30 \mathrm{~min}$ in a refrigerated centrifuge at $4{ }^{\circ} \mathrm{C}$ and $2000 \mathrm{~g}$. Platelet-poor plasma was transferred to small plastic vials, frozen in liquid nitrogen and stored at $-70^{\circ} \mathrm{C}$ until analysed. For the assay of C-reactive protein (CRP), serum samples were frozen in liquid nitrogen and stored at $-70^{\circ} \mathrm{C}$ until analysed.

Serum CRP levels were determined using an immunonephelometric method (N Latex High Sensitivity CRPTM, Dade Behring GmbH, Liederbach, Germany). Fibrinogen was assessed by the Clauss clotting method (Multifibren* U, Dade Behring, Marburg, Germany). ${ }^{17}$ Blood glucose concentration and lipid profile were determined using routine enzymatic methods and LDL cholesterol was calculated using the Friedewald formula. ${ }^{18}$ An enzymelinked immunosorbent assay was used for the measurement of plasma ox-LDL (Mercodia Oxidized LDL ELISA, Mercodia AB, Uppsala, Sweden).

\section{Statistical analysis}

The SPSS 15.0 statistical package for Windows (SPSS Inc., Chicago, IL, USA) was used for statistical analyses. The distribution of variables was tested with the Kolmogorov-Smirnov test. Variables with a normal distribution were shown as means and standard deviations, and differences between the groups were tested with the Student's $t$-test for independent samples or analysed by one-way analysis of variance (ANOVA). Since patient and control groups were not comparable regarding brachial artery diameter, the brachial artery diameter was adjusted for in further analysis where appropriate. Differences in FMD between the groups were analysed by one-way analysis of covariance (ANCOVA), with brachial artery diameter as a covariate. Variables with a non-normal distribution were shown as medians with ranges between the first and the third quartiles, while differences between two groups were tested with the Mann-Whitney rank sum test. The relationships between two cate- gorical variables were tested with the chi-squared test. For correlation analysis, Pearson's correlation coefficient was calculated for variables with normal distribution, and Spearman's rank-correlation coefficient for variables with non-normal distribution. Repeated measures of FMD were analysed by two-way mixed ANCOVA, with time of measurement as a repeated measure, the presence of symptomatic PAD as a between-groups variable, and baseline brachial artery diameter as a covariate. Repeated measures of brachial artery diameter and flow were analysed by two-way mixed ANOVA, with time of measurement as a repeated measure and the presence of symptomatic PAD as a between-groups variable. A two-tailed value of $p<0.05$ was considered statistically significant.

\section{Results}

\section{Clinical and biochemical characteristics}

Baseline characteristics of patients and controls are shown in Table 1. We found a past smoking history, hyperlipoproteinaemia and untreated mild or moderate arterial hypertension more frequent in patients than in controls. Out of $28(93 \%)$ patients with hyperlipoproteinaemia, $23(82 \%)$ were insufficiently treated.

All participants completed the study without any serious adverse events. Except for a longer period since cessation of smoking, higher ABI, and lower serum triglycerides and plasma ox-LDL in controls, there were no significant differences in clinical and biochemical characteristics between patients and controls (Table 2). Plasma ox-LDL was significantly higher in patients $\left(55.8 \pm 17.1 \mathrm{U} \mathrm{l}^{-1}\right.$ vs $6.3 \pm$ $1.6 \mathrm{U}^{-1}$ in controls, $p<0.001$ ), and in patients was negatively correlated with time since smoking cessation $(r=-0.41, p=0.027)$.

In patients, the median walking distance up to the onset of intermittent claudication was $82 \mathrm{~m}$ (interquartile range 59-112 m), and the median value of maximal walking distance was $220 \mathrm{~m}$ (interquartile range $120-471 \mathrm{~m}$ ). The leg with the lowest ABI was always the leg with the limiting claudication.

Of 30 patients included, $20(67 \%)$ were receiving statins, and among the latter only $5(25 \%)$ were achieving target lipid values according to NCEP ATP III Guidelines. Serum lipid values were lower in patients with statin treatment, most prominently LDL cholesterol $\left(2.45 \pm 0.73 \mathrm{mmol} \mathrm{l}^{-1}\right.$ vs $3.16 \pm$ $1.42 \mathrm{mmol} \mathrm{l}^{-1}$ in patients without statin treatment, $p=0.079$ ). However, we found no significant differences in baseline clinical and biochemical characteristics between patients with or without statin treatment (data not shown). 
Table 1 Baseline characteristics of PAD patients and controls

\begin{tabular}{lccc}
\hline Parameter & Patients $(n=30)$ & Controls $(n=14)$ & $p$-value \\
\hline Age (years) & $60.6(54.9-62.6)$ & $57.8(52.4-63.0)$ & 0.640 \\
Smoking history & 0 & 0 & \\
$\quad$ Current smokers & $29(97 \%)$ & $6(43 \%)$ & $<.001$ \\
$\quad$ Former smokers & $2.0(1.0-6.0)$ & $15.5(12.2-19.7)$ & 0.184 \\
Time since smoking cessation (years) & 0 & 0 & \\
Diabetes & $14(47 \%)$ & $9(21 \%)$ & \\
Arterial hypertension & $28(93 \%)$ & $30(100 \%)$ & 0 \\
Hyperlipoproteinaemia & $30(100 \%)$ & 0 & \\
Medications & $6(20 \%)$ & 0 & \\
Acetylsalicylic acid & $20(67 \%)$ & & \\
Pentoxifylline & Statin & & \\
\hline
\end{tabular}

Data are presented as a number (percentage) or as a median value and interquartile range. The $p$-value refers to comparison of patients and controls by the Mann-Whitney rank-sum test or chi-squared test, where feasible.

PAD, peripheral arterial disease.

\section{Haemodynamic measurements}

Before performing ultrasound measurements of vascular function, we investigated the morphology of the examined brachial artery segment. We found no disease-induced changes of the arterial wall structure and no indirect signs of the involvement of proximal brachial artery disease (e.g. pathological blood flow pattern) in any participant.

Values of resting brachial artery diameter were significantly lower in patients $(4.41 \pm 0.51 \mathrm{~mm}$ vs $4.80 \pm 0.37 \mathrm{~mm}$ in controls, $p=0.015)$. But there was no significant difference in hyperaemic brachial artery flow between both the groups studied (303 \pm

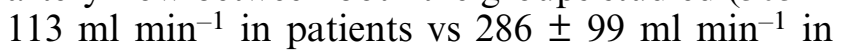
controls, $p=0.648$ ). In comparison with healthy controls, patients had borderline diminished capa- bility of baseline FMD $(7.03 \pm 1.99 \%$ vs $8.22 \pm$ $1.60 \%, p=0.057)$. This difference was significant after adjustment for brachial artery diameter $(p=0.009)$. The baseline FMD value was higher in patients with statin treatment, but the difference between patients with or without statin treatment was not significant $(7.24 \pm 1.81 \%$ vs $6.60 \pm 2.36 \%$ in patients without statin treatment, $p=0.413$ ).

\section{Vasodilator function after intermittent claudication \\ The values of resting brachial artery diameter, heart rate and blood pressure did not change significantly between the repeated FMD measurements. Hyperae- mic brachial artery flow did not change significantly after exercise in patients, but it was significantly}

Table 2 Clinical and biochemical characteristics of PAD patients and controls

\begin{tabular}{|c|c|c|c|}
\hline Parameter & Patients $(n=30)$ & Controls $(n=14)$ & $p$-value \\
\hline Age (years) & $60.6(54.9-62.6)$ & $57.8(52.4-63.0)$ & 0.640 \\
\hline Body mass index $\left(\mathrm{kg} \mathrm{m}^{-2}\right)$ & $26.3 \pm 2.6$ & $27.7 \pm 3.2$ & 0.130 \\
\hline \multicolumn{4}{|l|}{ Blood pressure } \\
\hline Systolic $(\mathrm{mmHg})$ & $141 \pm 16$ & $134 \pm 14$ & 0.119 \\
\hline Diastolic $(\mathrm{mmHg})$ & $84 \pm 9$ & $79 \pm 7$ & 0.064 \\
\hline \multicolumn{4}{|l|}{$\mathrm{ABI}$} \\
\hline Baseline & $0.58(0.51-0.81)$ & $1.05(0.96-1.18)$ & $<0.001$ \\
\hline 0 min after exercise & $0.24(0.17-0.45)$ & $1.12(1.05-1.25)$ & $<0.001$ \\
\hline 5 min after exercise & $0.46(0.26-0.71)$ & $1.06(1.00-1.17)$ & $<0.001$ \\
\hline Blood glucose $\left(\mathrm{mmol} \mathrm{I}^{-1}\right)$ & $5.58 \pm 0.65$ & $5.28 \pm 0.71$ & 0.174 \\
\hline \multicolumn{4}{|l|}{ Cholesterol } \\
\hline Total (mmol I-1) & $5.01 \pm 0.96$ & $4.86 \pm 0.81$ & 0.607 \\
\hline $\mathrm{HDL}\left(\mathrm{mmol} \mathrm{I}{ }^{-1}\right)$ & $1.27 \pm 0.51$ & $1.29 \pm 0.21$ & 0.878 \\
\hline $\operatorname{LDL}\left(\mathrm{mmol} \mathrm{I}{ }^{-1}\right)$ & $2.68 \pm 1.04$ & $2.94 \pm 0.65$ & 0.412 \\
\hline Triglycerides (mmol $\left.\mathrm{I}^{-1}\right)$ & $1.71(1.06-2.83)$ & $1.08(0.84-1.46)$ & 0.044 \\
\hline Fibrinogen $\left(\mathrm{g} \mathrm{I}^{-1}\right)$ & $3.25 \pm 0.95$ & $2.95 \pm 0.61$ & 0.283 \\
\hline $\operatorname{CRP}\left(\mathrm{mg} \mathrm{I}^{-1}\right)$ & $1.57 \pm 1.13$ & $1.43 \pm 1.24$ & 0.712 \\
\hline$o x-L D L\left(U^{-1}\right)$ & $55.8 \pm 17.1$ & $6.3 \pm 1.6$ & $<0.001$ \\
\hline
\end{tabular}

Data are presented as mean values \pm SD or as median values and interquartile range. The $p$-value refers to comparison of patients and controls by the independent $t$-test or Mann-Whitney rank-sum test.

$P A D$, peripheral arterial disease; $A B I$, ankle-brachial index; $H D L$, high-density lipoprotein; LDL, low-density lipoprotein; CRP, C-reactive protein; ox-LDL, oxidatively modified low-density lipoprotein. 
Table 3 Haemodynamic measurements at baseline and after exercise (at $30 \mathrm{~min}, 2 \mathrm{~h}$ and $4 \mathrm{~h}$ ) in patients ( $n=30)$ and controls $(n=14)$

\begin{tabular}{|c|c|c|c|c|c|}
\hline Parameter & Baseline & $30 \mathrm{~min}$ & $2 \mathrm{~h}$ & $4 \mathrm{~h}$ & $p$-value \\
\hline \multicolumn{6}{|c|}{ Resting brachial artery diameter (mm) } \\
\hline Patients & $4.41 \pm 0.51$ & $4.42 \pm 0.53$ & $4.42 \pm 0.55$ & $4.41 \pm 0.51$ & \multirow[t]{2}{*}{0.847} \\
\hline Controls & $4.80 \pm 0.37$ & $4.81 \pm 0.34$ & $4.81 \pm 0.32$ & $4.82 \pm 0.36$ & \\
\hline \multicolumn{6}{|c|}{ Resting brachial artery flow (ml $\left.\mathrm{min}^{-1}\right)$} \\
\hline Patients & $62 \pm 31$ & $73 \pm 38^{*}$ & $56 \pm 40$ & $52 \pm 35^{*}$ & \multirow[t]{2}{*}{0.908} \\
\hline Controls & $56 \pm 32$ & $63 \pm 22$ & $52 \pm 29$ & $45 \pm 27$ & \\
\hline \multicolumn{6}{|c|}{ Hyperaemic brachial artery flow (ml $\left.\mathrm{min}^{-1}\right)$} \\
\hline Patients & $303 \pm 113$ & $278 \pm 138$ & $276 \pm 115$ & $294 \pm 112$ & \multirow[t]{2}{*}{0.010} \\
\hline Controls & $286 \pm 99$ & $340 \pm 106^{*}$ & $367 \pm 124^{*}$ & $350 \pm 124^{*}$ & \\
\hline \multicolumn{6}{|l|}{ FMD (\%) } \\
\hline Patients & $7.03 \pm 1.99$ & $3.92 \pm 1.78 *$ & $6.36 \pm 2.12^{*}$ & $7.03 \pm 1.99$ & \multirow[t]{2}{*}{$<0.001$} \\
\hline Controls & $8.22 \pm 1.60$ & $8.66 \pm 1.88$ & $8.64 \pm 1.76$ & $8.64 \pm 1.57$ & \\
\hline
\end{tabular}

Data are presented as mean values \pm SD. The $p$-value refers to comparison of response difference in patients and controls by ANOVA for repeated measurements. ${ }^{*} p<0.05$ in comparison with the baseline values.

FMD, flow-mediated vasodilation.

increased after exercise in controls (Table 3). However, the difference in hyperaemic brachial artery flow between both the groups studied was significant only 2 hours after exercise $\left(276 \pm 115 \mathrm{ml} \mathrm{min}^{-1}\right.$ in

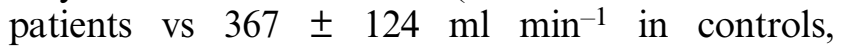
$p=0.021)$.

FMD decreased at $30 \mathrm{~min}$ after exercise and then gradually increased to its baseline value in patients, contrary to a slight non-significant increase after exercise in controls (Figure 1). The difference in FMD change between patients and controls over time was significant $(\mathrm{F}(1.86,76.27)=33.63, p<0.001)$. Comparing each point in time after exercise with the baseline, we found significant differences in FMD change between patients and controls at $30 \mathrm{~min}(\mathrm{~F}(1,41)=$ $57.68, p<0.001), 2 \mathrm{~h}(\mathrm{~F}(1,41)=7.55, p=0.009)$ and $4 \mathrm{~h}(\mathrm{~F}(1,41)=4.79, p=0.034)$ after exercise. The

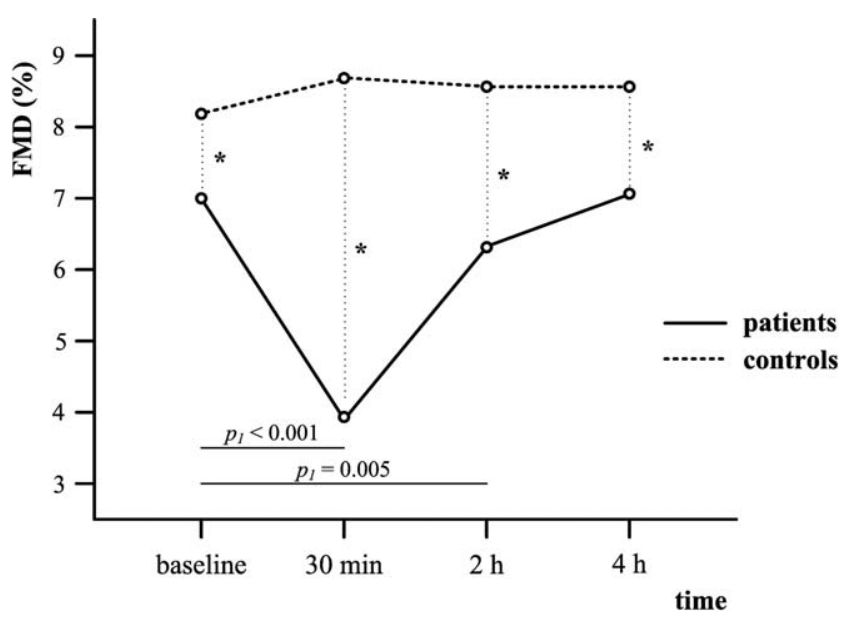

Figure 1 Flow-mediated vasodilation (FMD) at baseline and after exercise (at $30 \mathrm{~min}, 2 \mathrm{~h}$ and $4 \mathrm{~h}$ ) in patients $(n=30)$ and controls $(n=14)$. Data are presented as mean values, for clearer visualization without error bars. ${ }^{*} p<0.01$ between patients and controls. $p_{1}$, compared with baseline in patients. detailed results of further analysis are shown in Figure 1 and Table 3 . In comparison with its baseline value, FMD was significantly decreased in patients at $30 \mathrm{~min}(p<0.001)$ and at $2 \mathrm{~h}$ after exercise $(p=0.005)$. In comparison with controls, the FMD value was significantly lower in patients at $30 \mathrm{~min}$ $(p<0.001), 2 \mathrm{~h}(p<0.001)$ and $4 \mathrm{~h}$ after exercise $(p=0.002)$.

\section{Analysis of correlations between FMD and different parameters}

The correlation coefficients between FMD values and different parameters in all participants are shown in Table 4 . In all participants together, the baseline FMD was significantly negatively related to age $(r=-0.41, p=0.006)$ and arterial hypertension $(r=-0.36, p=0.017)$. We also found significant correlations between FMD values after exercise (at $30 \mathrm{~min}$ or $2 \mathrm{~h}$ ) and the presence of clinically manifest PAD, arterial hypertension, ABI, maximal walking distance, baseline FMD and plasma ox-LDL. There were no significant correlations between FMD values and time since smoking cessation, serum lipids, blood glucose and CRP.

\section{Discussion}

The present study describes the association of acute ischaemia during intermittent claudication in PAD patients with systemic vasodilator function over the course of several hours. This issue is of importance in view of the existing interest in prescribing physical training as a crucial part of the treatment of patients with atherosclerotic vascular disease, ${ }^{19}$ especially PAD. ${ }^{9,20}$ There is evidence of the long-term beneficial effects of physical activity in PAD patients. $7,8,20$ In contrast, some studies ${ }^{4,5}$ have suggested that 
Table 4 Correlations between FMD values (baseline, at $30 \mathrm{~min}$ and $2 \mathrm{~h}$ after exercise) and different parameters in all individuals $(n=44)$

\begin{tabular}{llll}
\hline & Baseline FMD & FMD at 30 min & FMD at $2 \mathrm{~h}$ \\
\hline Age & $-0.41^{* *}$ & -0.27 & -0.26 \\
PAD & -0.29 & $-0.78^{* *}$ & $-0.47^{* *}$ \\
Arterial hypertension & $-0.36^{*}$ & $-0.32^{*}$ & -0.29 \\
Baseline ABI & 0.29 & $0.56^{* *}$ & $0.33^{*}$ \\
ABI 0 min after exercise & 0.23 & $0.56^{* *}$ & $0.30^{*}$ \\
ABI 5 min after exercise & 0.22 & $0.50^{* *}$ & 0.24 \\
Maximal walking distance & 0.15 & $0.49^{* *}$ & $0.34^{*}$ \\
Baseline FMD & - & $0.68^{* *}$ & $0.85^{* *}$ \\
Plasma ox-LDL & -0.27 & $-0.67^{*}$ & $-0.40^{* *}$ \\
\hline
\end{tabular}

Results are presented as Pearson's correlation coefficients or as Spearman's rank-correlation coefficients. ${ }^{*} p \leq 0.05$, ** $p \leq 0.01$.

$P A D$, clinically manifest peripheral arterial disease; $A B I$, ankle-brachial index; FMD, flow-mediated vasodilation; oxLDL, oxidatively modified low-density lipoprotein.

exercise-induced transitory leg ischaemia in these patients leads to a release of anaerobic metabolites and their products to the circulation during the reperfusion period, with potentially harmful systemic effects. However, the results of the latter studies could be biased by the influence of medications and concomitant symptomatic atherosclerotic vascular disease of other locations in some participants, as well as the essential differences in the expression of some atherosclerotic risk factors between patients and controls (current smoking status, diabetes).

Impaired vascular function has been recognized as the earliest measurable change of the arterial wall in atherogenesis ${ }^{16}$ and a predictor of future cardiovascular events in patients with atherosclerotic vascular disease. ${ }^{21}$ In the present study, we performed the widely used non-invasive ultrasound measurement of $\mathrm{FMD}^{16}$ for studying vasodilator function. It was derived from previous observations that FMD additionally decreased in PAD patients shortly after exercise-induced maximal tolerable leg ischaemia. 5,6 To assess the duration of this phenomenon, we extended the observation time to 4 hours. Therefore, we followed the time course of changes in FMD of the clinically unaffected brachial artery after acute exercise-induced leg ischaemia in PAD patients.

As expected, we found significantly lower FMD values in PAD patients throughout the study. Our results further showed decreased FMD in PAD patients $30 \mathrm{~min}$ after exercise, followed by its gradual increase back to the baseline value at 4 hours after exercise, while in controls no significant changes of FMD were observed over time (Figure 1). A transitory decrease of FMD occurred in PAD patients after intermittent claudication in the absence of significant changes in hyperaemic flow stimulus and resting vessel diameter. This indicates the link between acute exercise-induced ischaemia and vasodilator function. The gradual improvement of FMD after cessation of ischaemic stimulus in PAD patients could be achieved through activation of adaptational mechanisms responsible for NO release and vascular function.

This study demonstrated non-significantly higher baseline FMD values in a subgroup of PAD patients with statin treatment. However, in view of the relatively small number of patients in each subgroup (with and without statin treatment), these results do not exclude a more impaired vasodilator function in patients without statin treatment, as indicated in recent studies. ${ }^{22,23}$ Also, this discrepancy between previous studies and the present results might partly be attributed to undertreatment with statins, leading to not entirely recognized statin effects in our PAD patients. Considering the NCEP ATP III Guidelines, ${ }^{24}$ the majority of PAD patients in the present study had insufficiently treated hyperlipoproteinaemia.

The baseline FMD values observed in our study in PAD patients were comparable with those in other studies performed in this clinical setting. ${ }^{21,25}$ On the contrary, baseline FMD values in the controls were lower than those observed in many other studies,${ }^{5,25}$ although still significantly higher than in PAD patients.

The lower FMD values observed in controls in the present study could be explained by the presence of some atherosclerotic risk factors, particularly age (over 40 years). ${ }^{26}$ Vascular dysfunction is considered as a forerunner of atherosclerotic presentations of cardiovascular disease, ${ }^{10}$ preceding other measurable changes in atherogenesis. ${ }^{27}$ As ABI probably has insufficient sensitivity for the detection of early atherosclerotic changes in the arterial wall, ${ }^{28}$ it could be assumed that perhaps initial subclinical atherosclerosis was already present in these controls. This assumption is further supported by the lack of significant differences in CRP and fibrinogen levels 
between the PAD patients and controls in this study, as higher levels of both inflammatory markers were demonstrated in atherosclerotic vascular disease in previous studies..$^{29,30}$ To avoid the possible influence of acetylsalicylic acid on inflammatory markers in this study, it was introduced temporarily in controls than run the risk of potential vascular events after its withdrawal in PAD patients. ${ }^{31}$

The expression of atherosclerotic risk factors was partially unbalanced in both examined groups. Arterial hypertension was more frequent in PAD patients than in controls, but further analysis revealed no significant differences in the absolute values of systolic and diastolic blood pressure between the two groups. However, we could not avoid the shorter time since smoking cessation and higher serum triglycerides in PAD patients.

A significantly higher plasma ox-LDL was demonstrated in PAD patients, associated with time since smoking cessation. This finding is in line with the results of a previous study that reported increased circulating ox-LDL in atherosclerotic vascular disease, also at the subclinical stage. ${ }^{32}$ Furthermore, in patients with atherosclerotic risk factor hypercholesterolemia, plasma ox-LDL was found to be associated with vascular function. ${ }^{33}$ In general, plasma ox-LDL in the present study was associated with FMD values throughout the study, considering all individuals (patients and controls) investigated together. Since patients with coronary artery disease and higher oxidative stress and impaired vascular function were observed to be particularly prone to future cardiovascular events, ${ }^{34}$ it can be presumed that the additional decrease in FMD associated with intermittent claudication, although transitory and of short duration, may be responsible for the progression of and the more advanced stage of atherosclerosis in PAD patients.

\section{Study limitations}

PAD patients with diabetes mellitus were not included in the study due to their expected (at least subclinical) neuropathy, ${ }^{35}$ with consequent disturbance in the perception of pain and/or possible different vascular response. Furthermore, we omitted FMD measurement immediately after exercise, since the expected increase in baseline brachial artery diameter at that time might result in a decreased additional FMD response during reactive hyperaemia as a consequence of systemic vasodilation caused by exercise.

\section{Conclusion}

This study shows that in PAD patients ischaemia during intermittent claudication is related to a transitory decrease of FMD of the clinically unaffected distant brachial artery. These findings indicate that acute local exercise-induced ischaemia is associated with transitory generalized functional deterioration of the arterial wall. Repeated local ischaemic events during intermittent claudication, associated with potentially harmful systemic effects, might explain the more advanced and generalized nature of atherosclerotic disease in PAD patients.

\section{Acknowledgements}

This study was supported by the Slovenian Research Agency, grant No. P3-0308. We would like to thank research nurses Simona Auman and Katja Janša-Trontelj and our laboratory staff for their expert assistance, and the Clinical Institute of Clinical Chemistry and Biochemistry at the University Medical Centre in Ljubljana for CRP measurements.

\section{References}

1 Ouriel, K. Peripheral arterial disease. Lancet 2001; 358: $1257-1264$.

2 Brevetti, G, Piscione, F, Silvestro, A, et al. Increased inflammatory status and higher prevalence of three-vessel coronary artery disease in patients with concomitant coronary and peripheral atherosclerosis. Thromb Haemost 2003; 89: 1058-1063.

3 Brevetti, G, De Caterina, M, Martone, VD, et al. Exercise increases soluble adhesion molecules ICAM-1 and VCAM1 in patients with intermittent claudication. Clin Hemorheol Microcirc 2001; 24: 193-199.

4 Signorelli, SS, Mazzarino, MC, Di Pino, L, et al. High circulating levels of cytokines (IL-6 and TNF $\alpha$ ), adhesion molecules (VCAM-1 and ICAM-1) and selectins in patients with peripheral arterial disease at rest and after a treadmill test. Vasc Med 2003; 8: 15-19.

5 Silvestro, A, Scopacasa, F, Oliva, G, de Cristofaro, T, Iuliano, L, Brevetti, G. Vitamin C prevents endothelial dysfunction induced by acute exercise in patients with intermittent claudication. Atherosclerosis 2002; 165: 277-283.

6 Silvestro, A, Schiano, V, Bucur, R, Brevetti, G, Scopacasa, $\mathrm{F}$, Chiariello, M. Effect of propionylcarnitine on changes in endothelial function and plasma levels of adhesion molecules induced by acute exercise in patients with intermittent claudication. Angiology 2006; 57: 145-154.

7 Brendle, DC, Joseph, LJO, Corretti, MC, Gardner, AW, Katzel, LI. Effects of exercise rehabilitation on endothelial reactivity in older patients with peripheral arterial disease. Am J Cardiol 2001; 87: 324-329.

8 Garg, PK, Tian, L, Criqui, MH, et al. Physical activity during daily life and mortality in patients with peripheral arterial disease. Circulation 2006; 114: 242-248.

9 Gardner, AW, Poehlman, ET. Exercise rehabilitation programs for the treatment of claudication pain: a metaanalysis. JAMA 1995; 274: 975-980. 
10 Gonzalez, MA, Selwyn, AP. Endothelial function, inflammation, and prognosis in cardiovascular disease. Am J Med 2003; 115: 99S-106S.

11 Silvestro, A, Scopacasa, F, Ruocco, A, et al. Inflammatory status and endothelial function in asymptomatic and symptomatic peripheral arterial disease. Vasc Med 2003; 8: 225 232.

12 Celermajer, DS, Sorensen, KE, Bull, C, Robinson, J, Deanfield, JE. Endothelium-dependent dilation in the systemic arteries of asymptomatic subjects relates to coronary risk factors and their interaction. J Am Coll Cardiol 1994; 24: $1468-1474$.

13 Healy, B. Endothelial cell dysfunction: an emerging endocrinopathy linked to coronary disease. $\mathrm{J} \mathrm{Am}$ Coll Cardiol 1990; 16: 357-358.

14 Poredoš, P, Kek, A. Relation of blunted dilation of the brachial artery in insulin-dependent diabetes mellitus to microalbuminuria. Am J Cardiol 2000; 86: 364-367.

15 Fontaine, R, Kim, M, Kieny, R. Die chirurgische behandlung der peripheren durchblutungsstörungen. Helv Chir Acta 1954; 5/6: 499-533.

16 Celermajer, DS, Sorensen, KE, Gooch, VM, et al. Noninvasive detection of endothelial dysfunction in children and adults at risk of atherosclerosis. Lancet 1992; 340: 1111-1115.

17 Clauss, A. Gerinnungsphysiologische Schnellmetode zur Bestimmung des Fibrinogens. Acta Haemat 1957; 17: 237 246.

18 Friedewald, WT, Levy, RI, Fredrickson, DS. Estimation of the concentration of low-density lipoprotein cholesterol in plasma, without use of the preparative ultracentrifuge. Clin Chem 1972; 18: 499-502.

19 Ignarro, LJ, Balestrieri, ML, Napoli, C. Nutrition, physical activity, and cardiovascular disease: an update. Cardiovasc Res 2007; 73: 326-340.

20 Stewart, KJ, Hiatt, WR, Regensteiner, JG, Hirsch, AT. Exercise training for claudication. $N$ Engl J Med 2002; 347: 1941-1951.

21 Brevetti, G, Silvestro, A, Schiano, V, Chiariello, M. Endothelial dysfunction and cardiovascular risk prediction in peripheral arterial disease. Additive value of flowmediated dilation to ankle-brachial pressure index. Circulation 2003; 108: 2093-2098.

22 Jarvisalo, MJ, Toikka, JO, Vasankari, T, et al. HMG CoA reductase inhibitors are related to improved systemic endothelial function in coronary artery disease. Atherosclerosis 1999; 147: 237-242.

23 Taneva, E, Borucki, K, Wiens, L, et al. Early effects on endothelial function of atorvastatin $40 \mathrm{mg}$ twice daily and its withdrawal. Am J Cardiol 2006; 97: 1002-1006.
24 Grundy, SM, Cleeman, JI, Bairey Merz, CN, et al. Implications of recent clinical trials for the National Cholesterol Education Program Adult Treatment Panel III Guidelines. Circulation 2004; 110: 227-239.

25 Loffredo, L, Marcoccia, A, Pignatelli, P, et al. Oxidativestress-mediated arterial dysfunction in patients with peripheral arterial disease. Eur Heart J 2007; 28: 608-612.

26 Celermajer, DS, Sorensen, KE, Spiegelhalter, DJ, Georgakopoulos, D, Robinson, J, Deanfield, JE. Aging is associated with endothelial dysfunction in healthy men years before the age-related decline in women. $\mathrm{J} \mathrm{Am}$ Coll Cardiol 1994; 24: 471-476.

27 Poredoš, P. Endothelial dysfunction and cardiovascular disease. Pathophysiol Haemost Thromb 2002; 32: 274-277.

28 McDermott, MM, Liu, K, Criqui, MH, et al. Ankle-brachial index and subclinical cardiac and carotid disease. The multi-ethnic study of atherosclerosis. Am J Epidemiol 2005; 162: $33-41$.

29 Brevetti, G, Silvestro, A, Di Giacomo, S, et al. Endothelial dysfunction in peripheral arterial disease is related to increase in plasma markers of inflammation and severity of peripheral circulatory impairment but not to classic risk factors and atherosclerotic burden. J Vasc Surg 2003; 38: 374-379.

30 Ridker, PM, Stampfer, MJ, Rifai, N. Novel risk factors for systemic atherosclerosis. A comparison of C-reactive protein, fibrinogen, homocysteine, lipoprotein(a), and standard cholesterol screening as predictors of peripheral arterial disease. JAMA 2001; 285: 2481-2485.

31 Blinc, A, Poredoš, P. Pharmacological prevention of atherothrombotic events in patients with peripheral arterial disease. Eur J Clin Invest 2007; 37: 157-164.

32 Holvoet, P, Jenny, NS, Schreiner, PJ, Tracy, RP, Jacobs, DR. for the Multi-Ethnic Study of Atherosclerosis. The relationship between oxidized LDL and other cardiovascular risk factors and subclinical CVD in different ethnic groups: The Multi-Ethnic Study of Atherosclerosis (MESA). Atherosclerosis 2007; 194: 245-252.

33 Matsuoka, H. Endothelial dysfunction associated with oxidative stress in human. Diabetes Res Clin Pract 2001; 54: S65-S72.

34 Heitzer, T, Schlinzig, T, Krohn, K, Meinertz, T, Munzel, T. Endothelial dysfunction, oxidative stress, and risk of cardiovascular events in patients with coronary artery disease. Circulation 2001; 104: 2673-2678.

35 Boulton, AJM, Vinik, AI, Arezzo, JC, et al. Diabetic neuropathies. A statement by the American diabetes association. Diabetes Care 2005; 28: 956-962. 